\title{
Análise do campo conceitual da engenharia de sistemas cognitivos e proposta de uma nova agenda de pesquisa
}

\author{
Eder Henriqson $^{\text {a* }}$, Tarcisio Abreu Saurin ${ }^{\mathrm{b}}$ \\ a*ehenriqson@pucrs.br, PUCRS, Brasil \\ bsaurin@ufrgs.br, UFRGS, Brasil
}

\begin{abstract}
Resumo
Este artigo tem por objetivo descrever a evolução do campo conceitual da Engenharia de Sistemas Cognitivos (ESC), discutir seus fundamentos epistemológicos e verificar a sua coerência com a natureza dos problemas investigados. Para tanto, o trabalho apresenta uma revisão bibliográfica do desenvolvimento histórico desse campo e a caracterização de cinco abordagens comuns nas pesquisas em ESC. Os resultados apontam que os estudos em ESC, via de regra, tomam o indivíduo ou o sistema técnico como unidade de análise, deixando lacunas importantes na investigação de interações entre humano, trabalho e artefatos. Uma nova agenda de pesquisa é proposta tendo como base três premissas: um sistema cognitivo não deve ser interpretado como uma máquina cartesiano-newtoniana, que possui relações evidentes de causa e efeito; um sistema cognitivo não pode ser modelado com precisão e perfeição; a investigação de sistemas cognitivos deve focar em fenômenos emergentes das interações entre humano, trabalho e artefatos tecnológicos.
\end{abstract}

Palavras-chave

Engenharia de sistemas cognitivos. Sistemas sociotécnicos complexos. Possibilidades de agenda de pesquisa.

\section{Introdução}

A engenharia de sistemas cognitivos (ESC) tem se legitimado como disciplina científica desde meados da década de 1980. Desde então, três períodos de desenvolvimento da ESC podem ser identificados (WOODS; HOLLNAGEL, 2006): (a) o período de construção dos fundamentos conceituais, na década de 1980; (b) o período de formulação de métodos, ferramentas e de estudos aplicados, na década de 1990; e (c) o período da consolidação e legitimação do campo científico, na década de 2000.

0 período conceitual da década de 1980 foi caracterizado pelo desenvolvimento de teorias e métodos que permitissem a investigação do desempenho dos sistemas sociotécnicos sob a perspectiva desse novo campo. Esse período foi marcado, sobretudo, pela crítica ao modelo de ciência cognitiva da época, fortemente embasado na análise do desempenho humano por meio de experimentos cognitivos em laboratório (HOFFMAN; WOODS, 2000; McNEESE, 2002).

As concepções teóricas formadas na década de 1980, para a análise das interações humanomáquina em contextos complexos, criaram condições para o desenvolvimento de métodos e ferramentas de investigação na década de 1990. Esse período foi caracterizado pelo incremento significativo no número de pesquisas de campo, segundo essa nova abordagem, e pelo consequente desenvolvimento de métodos de análise e protocolos de pesquisa (RESING, 2002; HOLLNAGEL; WOODS, 2005).

$\mathrm{Na}$ esteira dessas duas décadas de produção, a ESC ganhou legitimidade científica como disciplina de fatores humanos nos últimos 10 anos (HOFFMAN et al., 2002; HOFFMAN; WOODS, 2000; SARTER; AMALBERTI, 2005). As consequências mais importantes têm sido: (a) a facilitação de acesso 
a campos de estudo, visando à investigação de contextos reais de trabalho; (b) o projeto centrado no elemento humano, assumindo que ele é o condutor primário de sistemas tecnológicos; (c) a investigação do impacto da introdução das novas tecnologias, sob uma perspectiva que considera conjuntamente a relação humano-trabalho-artefato.

0 foco da ESC no comportamento e na cognição trouxe como reconhecimento a crescente necessidade de investimentos na capacidade humana para lidar com sistemas complexos. Por outro lado, o foco no projeto de tecnologias contribuiu para promover a crença de que os projetistas deveriam projetar os artefatos à imagem da cognição humana (ver exemplos em ALBRECHTSEN et al., 2001; HOLLNAGEL, 1999). Todavia, ao fazer isso, as pesquisas em ESC adotam pressupostos fortemente vinculados aos modelos de cognição baseados na teoria de processamento da informação (Information Processing Theory - IPS). 0 IPS nivela as propriedades da cognição humana às propriedades funcionais dos dispositivos computacionais (por exemplo, memória, tempo de processamento) (NEWELL; SHAW; SIMON, 1958; HARRÉ, 1999; HATFIELD, 2002; MILLER, 1988). Como consequência, a perspectiva do IPS resulta em um modelo de representação da cognição fortemente vinculado ao modelo da máquina, com estruturas, funções, inter-relações evidentes, entre outros.

0 objetivo desta pesquisa é, pois, descrever de que modos o campo conceitual da ESC vem sendo constituído, explicitando seus pressupostos epistemológicos e verificando a coerência deles com a natureza dos problemas abordados pela ESC. Adicionalmente, este estudo apresenta a proposta de uma nova agenda de pesquisa em sistemas sociotécnicos complexos, a partir das inconsistências detectadas entre o que a literatura acerca da ESC defende e o que de fato vem sendo produzido pelos estudos empíricos.

Para tanto, o trabalho apresenta uma revisão bibliográfica do desenvolvimento histórico da ESC e a caracterização de abordagens comuns nas pesquisas. A revisão de literatura teve como foco trabalhos seminais (teóricos e aplicados), que apresentaram inovações teóricas ou metodológicas ligadas a pesquisadores que explicitamente vinculam seus trabalhos ao campo da ESC.

\section{As revoluções cognitivas}

A palavra cognição remonta aos escritos de Platão e Aristóteles. Todavia, foi na metade do século passado, mais precisamente nos anos 1950 e 1960 que dois movimentos marcaram o início do estudo da cognição, especificamente nas ciências sociais, chamados de primeira e segunda revolução cognitiva (BAARS, 1986; BEM; KEIJZER, 1996; MILLER, 2003; O'DONOHUE; FERGUSON; NAUGLE, 2003; SPERBER; HIRSCHFELD, 2001). Tais movimentos são caracterizados pela convergência de trabalhos teóricos e experimentais em diversas áreas, tais como filosofia, psicologia, linguística, neurociências, sociologia e antropologia.

\subsection{A primeira revolução cognitiva}

A primeira revolução cognitiva foi marcada pelo paralelismo estabelecido entre o funcionamento da máquina (inicialmente os rádios e posteriormente os computadores) e a atividade mental humana (MILLER, 2003; NEWELL, 1980; POSNER et al., 1988; SKINNER, 1989). 0 movimento surgiu como reação ao behaviorismo, tomando força no campo da psicologia e visando descortinar os fenômenos mentais para explicar a origem de determinados comportamentos. Estudos da cibernética e as teorias do processamento da informação, igualmente, alavancaram pesquisas laboratoriais, impulsionando a formação de enunciados e pressupostos científicos sobre a cognição aplicada.

Quando equipararam cognição com as teorias de processamento da informação, traçando um paralelismo entre a mente humana e os computadores (HOLLNAGEL; WOODS, 2005), a ideia fundamental dos pesquisadores era explicar o funcionamento de um software (a cognição) em um hardware (o cérebro e o sistema nervoso) (BEM; KEIJZER, 1996). 0 estruturalismo e a linearidade imbricados nessa forma de pensar foram responsáveis pela criação de uma série de modelos do funcionamento da mente humana. 0 paralelismo traçado com os computadores era tão evidente que ambos, modelos da mente e de computadores, compartilharam definições de elementos dessa estrutura (por exemplo, memória de longo prazo, memória volátil, tempo de processamento).

Motivados pelo estudo do desempenho humano e, principalmente, pela compreensão do erro humano, pesquisadores desenvolveram um corpo de ciência para legitimar a fragilidade e as limitações humanas na realização de tarefas complexas. Conceitos como consciência situacional (ver, por exemplo, ENDSLEY, 1995a, b; ENDSLEY; GARLAND, 2000), complacência (ver, por exemplo, PARASURAMAN; RILEY, 1997; PARASURAMAN, SHERIDAN; WICKENS, 2000), dinâmica atencional (ver, por exemplo, WICKENS; HOLLAND, 2000; WOODS et al., 2010), modelos mentais (ver JOHNSON-LAIRD, 1980; KIERAS; BOVAIR, 1984), carga de trabalho (ENDSLEY; KABER, 1999; HART, 1982; KABER; ENDSLEY, 2004; KABER; RILEY, 1999), tomada de decisão (WICKENS, 1992; WICKENS; HOLLNAD, 2000), tempo de resposta (HICK, 1952; 
HYMAN, 1953; McCLELLAND, 1979; McCULLOCH; PITTS, 1943; WICKENS; HOLLAND, 2000), entre outros, foram conceitos investigados em laboratório para explicar o comportamento humano.

Embora as teorias de processamento da informação, que marcaram o estudo da cognição na primeira revolução cognitiva, não tenham fornecido uma teoria abrangente e universalmente aceita acerca da cognição humana, os seus pressupostos têm servido como um arcabouço de ideias para a formulação de teorias cognitivas. Das teorias de processamento de informação surgiram e se consolidaram: (a) os modelos computacionais da mente (NEWELL; SHAW; SIMON, 1958); (b) a manutenção da prática dos experimentos em laboratório para análise da mente e dos processos mentais (WAGEMANS; VERSTRATEN; HE, 2001); e (c) a influência das pesquisas em neurociência (POSNER; DEHAENE, 1994; POSNER et al., 1988).

A ideia central das teorias do IPS é que a cognição funciona como um sistema de processamento da informação de entrada (input), operação dessa informação na mente (processing) e saída dessa informação (output) (NEWELL; SHAW; SIMON, 1958; RASMUSSEN; PEJTERSEN; GOODSTEIN, 1994). Os inputs ocorreriam por meio dos canais perceptivos (audição, tato, paladar, olfato, visão e equilíbrio), responsáveis por intermediar a relação do mundo externo com o mundo interno da mente do indivíduo. 0 processamento da informação poderia, então, ser explicado por um sistema de modelos de funcionalidade da mente, abrindo o que outrora foi considerado uma "caixa preta". Os outputs poderiam ser considerados como as respostas do humano (operador) no processo de interação entre e com o ambiente externo.

Exemplares dessa lógica de pensamento são os trabalhos de Wiener (1988) e Wiener e Curry (1980), que mapearam a relação humano-máquina na cabine de aeronaves, identificando circularidades (loopings) de informação entre o operador e a máquina. A visão desses autores pressupunha que toda ação do operador fosse mediada por um conjunto de símbolos representados pela máquina (output de um determinado estado), capturado pelo operador através da percepção (input), processado/raciocinado pelo operador por meio de uma série de categorias de conhecimento (prossessing), resultando numa ação de controle sobre a máquina (output). Mais tarde, essa mesma perspectiva foi modelada em simuladores de voo por Endsley e colaboradores (ENDSLEY, 1995a, b; ENDSLEY; GARLAND, 2000; ENDSLEY; KABER, 1999; ENDSLEY; KIRIS, 1995). Nesse caso, a "caixa preta" do processamento de informação da mente humana foi, supostamente, aberta pelo "descobrimento" de níveis de consciência situacional e a articulação conjunta de uma série de mecanismos da mente humana identificados pela psicologia cognitiva experimental como: memória, atenção, tempo de resposta, estrutura de conhecimentos e roteiros de ação. 0 passo seguinte foi a popularização desses conceitos para a identificação de erros e falhas dos operadores em algum dos níveis de consciência situacional, condição geralmente denominada de "perda de consciência situacional". Tais conceitos têm sido criticados e referenciados como folk models por alguns pesquisadores desse campo. Um debate aprofundado sobre essas críticas pode ser encontrado nos trabalhos de Dekker (2000), Dekker e Woods (2002), Dekker e Hollnagel (2004), Parasuraman, Sheridan e Wickens (2008) e Dekker et al. (2010).

\subsection{A segunda revolução cognitiva}

A segunda revolução cognitiva caracterizou-se pelo questionamento dos enunciados e pressupostos científicos da primeira revolução (HARRÉ, 1999; HUTCHINS, 1995a; LASZLO, 2004; MILLER, 2003). Mais precisamente, pesquisadores de diferentes áreas passaram a questionar: (a) a teoria do processamento da informação como paradigma de compreensão dos fenômenos cognitivos (por exemplo, RASMUSSEN, 1985); (b) a validade ecológica dos achados científicos da primeira revolução, fortemente embasados em evidências empíricas geradas por experimentação em ambientes de laboratório (por exemplo, WOODS, 1993, 2003); e (c) o deslocamento do estudo da cognição da mente para o ambiente, destacando as dimensões culturais e interacionistas da relação entre humano e ambiente (por exemplo, HUTCHINS, 1995a, b).

Validade ecológica representa similaridade entre as condições da pesquisa e as condições reais que se deseja conhecer por meio da pesquisa (GIBBSON, 1979). Representa, portanto, a medida da aproximação da pesquisa ao mundo real e está associada à validade externa e à generalização (WOODS, 2003). Para os teóricos da segunda revolução, a ideia da cognição como algo interno à mente humana, envolvendo o processamento de símbolos e categorias e ativado pela interação do humano com o mundo exterior limita a compreensão da dinâmica do pensamento e da criação de significados (NEISSER, 1967, 1976; LASZLO, 2004).

Conceitos como carga de trabalho, complacência e tomada de decisão foram originados, testados e enunciados em ambiente artificiais. Estudos da relação entre a carga de trabalho e o desempenho humano frente a determinadas situações originaram enunciados, mais tarde, aceitos como "verdade", tais como "a curva $\mathrm{U}$ de desempenho versus carga de trabalho", originalmente proposta e testada em 
ratos de laboratório (YERKES; DODSON, 1908). A falta de validade ecológica dos enunciados científicos tornou-se alvo dos teóricos da segunda revolução.

Outro exemplo são as teorias da tomada de decisão. Diversos modelos para o estudo do processo de decisão foram desenvolvidos por teóricos da primeira revolução (ENDSLEY et al., 2007; POTTER et al., 2002). Tais modelos caracterizam-se, quase sempre, pela influência das teorias econômicas (racionalistas) da decisão (BURNS, 2007), as quais consideram que o decisor tem sempre a informação, tem sempre tempo para tomar a decisão, avalia as alternativas presentes e seleciona aquela mais adequada e monitora e avalia continuamente o curso de ação tomado. Esse olhar para as teorias do processo decisório acaba por desenvolver estratégias de treinamento para capacitação dos decisores (por exemplo, CRM na aviação), bem como por propor um conjunto de heurísticas para assessorar a tomada de decisão, tais como o modelo DECIDE (detect, estimate, choise, implement, diagnose, evaluate) recomendado pelo Federal Aviation Administration (FAA) nos Estados Unidos (FEDERAL..., 1991).

A abordagem ecológica da segunda revolução cognitiva põe ênfase nas relações do humano com o contexto (incluído trabalho e ambiente) de interação. Nessa visão, não há como separar uma mente individual de seu contexto sociocultural (HUTCHINS, 1995a; LOPES; LOPES; TEIXEIRA, 2004; NEISSER, 1967, 1976). Fazer isso “[...] eliminaria o caráter construtivo e não passivo do homem frente ao seu mundo e a si mesmo." (LOPES; LOPES; TEIXEIRA, 2004, p. 21).

Seguindo a lógica dos teóricos da segunda revolução cognitiva e impulsionando os estudos da cognição social, Hutchins (1995a, b) propõe que a cognição seja entendida como um fenômeno cultural e distribuído. Quando publica o artigo How a cockpit remembers its speeds (HUTCHINS, 1995a), o autor descreve o cockpit de uma aeronave e a cognição distribuída no mesmo para revelar, através de analogia com os mecanismos da memória da mente humana, como as velocidades de pouso são lembradas pelo cockpit. 0 autor descreve um sistema funcional presente na cabine que ativa diferentes modos de memória ao longo de um voo, demonstrando que a memorização das velocidades não é uma função exclusivamente interna da mente dos pilotos, mas um fenômeno distribuído também em mídias externas (por exemplo, take-off data card) do cockpit. Esse trabalho corrobora o conceito de sistema cognitivo (sistema formado pela coatuação metaorientada de humano, tecnologia e trabalho), muito embora a analogia ainda seja fortemente estruturalista para a explicação dos fenômenos da cognição distribuída.
É importante citar, também, que essa lógica de Hutchins para o estudo da cognição não foi a primeira abordagem dessa natureza, bem antes disso Piaget (1973) e Vygotsky (1978) já desenvolviam teorias de aprendizagem baseadas na cognição como um processo da interação entre sujeito e objeto.

Ao contrário da primeira revolução, a segunda passou a considerar as pessoas como seres não autônomos e intencionais, mas como partes de um contexto mais amplo no mundo natural. Os símbolos não são naturalmente encontrados no mundo e nem estão estampados na mente humana, eles são culturalmente fabricados.

\section{Como as teorias e os métodos em ESC vêm sendo constituídos?}

Nesta seção são apresentados cinco grupos caracterizados pelos pesquisadores-líderes no estudo da ESC nos dias atuais. Embora outros pesquisadores também exerçam liderança no estudo dos fenômenos cognitivos no campo da engenharia de fatores humanos (tais como R. Amalberti, C. Wickens, R. Parassuraman, T. Sheridan), a descrição dos grupos é delimitada aos pesquisadores que deliberadamente se identificam como vinculados ao campo da ESC em suas publicações. Os grupos representam o ponto de origem de inovações teóricas ou metodológicas no campo da ESC, usados por outros pesquisadores em maior escala.

A classificação adotada é inspirada no trabalho de Eggleston (2002) que, partindo do trabalho de Vicente (1998), descreveu quatro genótipos dos estudos em ESC. Ao contrário de Eggleston (2002) e Vicente (1998), a classificação aqui apresentada segue critérios de classificação diferenciados e com outros propósitos. A categorização dos cinco grupos aqui realizada não busca ser exaustiva ou excludente, mas sim propiciar um ponto de partida para discussão de características como: (a) principais representantes do grupo; (b) a abordagem geral de estudo da cognição, classificada segundo as teorias da primeira ou segunda revolução cognitiva; (c) o foco de análise dos trabalhos; (d) os principais domínios de aplicação; e (e) os pressupostos teóricos, modelos ou métodos desenvolvidos.

\subsection{Grupo $A$}

O grupo A teve origem na Carnegie Mellon University, nos Estados Unidos, por meio dos estudos liderados por Stwart Card e colaboradores, entre eles T. P. Moran, A. Newell (EGGLESTON, 2002). 0 objetivo fundamental dos trabalhos desse grupo era estudar o desempenho humano na interação humano-máquina 
em sistemas tecnológicos. A abordagem central da cognição tinha bases nas teorias de processamento de informação. Usando a lógica do processamento de informação, os pesquisadores objetivavam desenvolver modelos capazes de simular e quantificar o desempenho humano nas suas interações com os sistemas tecnológicos. A proposta era identificar constrangimentos do sistema e propor design de equipamentos mais eficazes a partir da avaliação do custo físico e cognitivo de interação com as tecnologias.

Nesse sentido, dois modelos foram propostos por esse grupo e foram logo difundidos: Model of Human Processor (MHP) (CARD; MORAN; NEWELL, 1980, 1983); e o Goals, Operators, Methods, Selections (GOMS) (CARD; MORAN; NEWELL, 1986). Ao desenvolver o MHP, os pesquisadores citados incluíram na arquitetura lógica dos processos de cognição o estágio perceptual e o de resposta motora para entendimento do desempenho humano. Algumas críticas localizam o MHP como um modelo de comportamento humano, pois sua abordagem não está circunscrita aos fenômenos internos da cognição (mente) humana (EGGLESTON, 2002). Todavia, o modelo ficou conhecido como uma das primeiras tentativas de transferir as descobertas da ciência cognitiva para a análise da interação humano-máquina.

0 GOMS representa uma abordagem de análise do trabalho ou da tarefa na perspectiva da cognição, segundo a lógica do processamento da informação, assim como o MHP. A ideia central do modelo é que todo trabalho humano consiste em uma série de tarefas necessárias para transformar um estado inicial em outro estado final desejado (meta). Para atingir essa meta caberia ao operador transformar o estado abstrato da meta em uma sequência de ações mentais e físicas. Para tanto, a pessoa deveria desenvolver e aplicar procedimentos relacionados à solução de problemas, delimitada pela estrutura do contexto da tarefa, do conhecimento humano e das capacidades e limitações de processamento.

Os principais campos de aplicação do desenvolvimento dos trabalhos desse grupo são a análise das interações entre humanos e computadores. Diversas variações do modelo GOMS, como Keystroke Level Modeling (OLSON; OLSON, 1990), Natural GOMS Language (BOVAIR; KIERAS; POLSON, 1990; KIERAS, 1988, 1996; KIERAS; POLSON, 1985) e CognitivePerceptual-Motor GOMS (GRAY; JOHN; ATWOOD, 1992) podem ser encontradas.

\subsection{Grupo $B$}

O grupo B teve origem na Universidade da Califórnia em San Diego, nos Estados Unidos, liderado por Donald Norman e colaboradores, entre eles D. G.
Borrow (EGGLESTON, 2002). Os pesquisadores desse grupo consideram a ESC como um campo para o desenvolvimento de teorias para orientar o projeto, construção e uso de sistemas tecnológicos. A cognição é tratada a partir da teoria do processamento da informação, à semelhança dos trabalhos desenvolvidos pelos representantes do grupo A, diferenciando-se pela ênfase dos resultados estarem vinculados ao design de sistemas tecnológicos.

A principal contribuição teórico-metodológica desse grupo é a proposição do modelo de Sete Estágios (Seven-stage Model) (NORMAN, 1986). 0 objetivo do modelo é conciliar as demandas físicas do sistema com as demandas psicológicas dos operadores. Assim como o GOMS, o Modelo de Sete Estágios busca caracterizar o comportamento humano no desempenho das tarefas, posicionando o humano como o agente do processamento de informações e resolução de problemas. A premissa é definir, a partir dessa análise, uma linguagem do trabalho e uma linguagem de interface entre o mundo psicológico do operador (atividade mental) e o mundo físico da tarefa e da tecnologia. Os estudos desse grupo focam-se no desenvolvimento de interfaces centradas no usuário, especialmente no âmbito das ferramentas (hardware e software) computacionais.

\subsection{Grupo $C$}

0 grupo C teve origem no RIS $\emptyset$ National Laboratory da Dinamarca, liderado por Jens Rasmussen e colaboradores, entre eles K. Vicente, A. M. Pejtersen, L. P. Goodstein, K. Shmidt (EGGLESTON, 2002). A abordagem geral do estudo da cognição, embora aplicada em contextos mais amplos do que no entendimento do funcionamento da mente humana, tais como a análise de processos organizacionais, segue pressupostos de linearidade fortemente embasados na teoria do processamento da informação. Paralelamente, os estudos são marcados, em geral, por maior validade ecológica, uma vez que são, preponderantemente, realizados em contextos reais.

Dentre os principais pressupostos teóricos, modelos ou métodos desenvolvidos por esse grupo destaca-se a proposição de uma estrutura para análise do trabalho (RASMUSSEN; PEJTERSEN; GOODSTEIN, 1994; RASMUSSEN; PEJTERSEN; SHMIDT, 1990). A estrutura fundamenta-se na investigação e compreensão de cinco grandes constructos: o domínio do trabalho, o controle das tarefas, o controle das estratégias, o contexto das interações sociais e as capacidades e limitações dos agentes.

A abstração de hierarquias e a análise das relações meio-fim são aplicadas como técnicas 
para descortinar as diferentes funções do domínio do trabalho (BISANTZ; VICENTE, 1994; NAIKAR; SANDERSON, 1999; NAIKAR; SANDERSON; LINTERN, 2000; RASMUSSEN, 1986; RASMUSSEN; PEJTERSEN; GOODSTEIN, 1994; VICENTE, 1999). 0 controle das tarefas é analisado com base nas necessidades adjacentes à meta de cada tarefa realizada, tomando como base a Decision Ladder Chart (RASMUSSEN, 1986), que analisa o controle das tarefas em termos de processamento da informação (também chamado de atividades cognitivas) e conhecimentos necessários por parte do decisor. No estágio seguinte, controle das estratégias, a análise das decisões é realizada com base nos mesmos pressupostos do estágio anterior, tendo, contudo, foco diferenciado na "estratégia". 0 contexto das interações sociais é investigado com base nas propriedades sociais e organizacionais do trabalho, incluindo a divisão do trabalho, estilos de liderança e processos de coordenação. As capacidades e limitações dos agentes (humanos e máquinas) são discutidas à luz dos constrangimentos criados pelas demais condições apresentadas nos construtos anteriores, evidenciando a forma como elas interferem na competência dos agentes.

Nesse sentido, Rasmussen (1985) desenvolveu, ainda, a taxonomia SRK (Skills-Rules-Knowledge), a qual consolida a visão do humano como processador de informação em um sistema e manipulador de símbolos, considerando diferentes situações de trabalho (situações de rotina, situações para quais os operadores são treinados e situações novas ou inusitadas) e diferentes modos de processamento da informação (por exemplo, consciente, misto ou automático). As situações de trabalho e os modos de processamento da informação requeridos em tais situações determinariam padrões de comportamento dos operadores baseados em habilidades, regras ou conhecimento, definindo o custo cognitivo da tarefa e oferecendo uma alternativa para a compreensão dos erros decorrentes de ação. Essa discussão é aprofundada, mais tarde, por Reason (1990), quando apresentar um protocolo para classificação de erros e violações.

0 foco geral desses métodos é a análise do trabalho, do design do sistema e do desempenho do operador. Com métodos mais prescritivos, os trabalhos do grupo $C$ rapidamente se difundiram, tendo como principal campo de atuação o segmento de geração de energia nuclear.

\subsection{Grupo $D$}

O grupo D teve como origem os estudos de David Woods e colaboradores (entre eles R. Cook, E. Hollnagel, S. Dekker e K. Christoffersen), na Ohio
State University, nos Estados Unidos. Baseados na premissa da cognição como um fenômeno distribuído no sistema e partindo para uma abordagem ecológica de investigação, o foco dos investigadores desse grupo é a análise de como os sistemas tecnológicos computacionais podem auxiliar o ser humano na tomada de decisão e execução do trabalho.

Em seus trabalhos, fortemente influenciados pelas ideias da segunda revolução cognitiva, em especial o conceito de cognição distribuída, Woods assume a atividade como o meio pelo qual humanos e artefatos coatuam (WOODS; HOLLNAGEL, 2006; WOODS et al., 2002). 0 autor defende que, mais do que inter-relação, humanos e artefatos (especialmente os chamados artefatos tecnológicos) são coautores da ação. Nesse sentido, a cognição posiciona-se no processo de interação entre as partes.

Em artigo intitulado Cognitive systems engineering: New wine in new bottles, Hollnagel e Woods (1983) propõem que a nova unidade de análise do trabalho deve ser o sistema cognitivo correlacionado. 0 vinho novo seria a perspectiva da cognição distribuída, num sentido mais amplo do que o estudo da cognição da mente, característico da primeira revolução. As garrafas novas referem-se à mudança do foco de análise do interacionismo da relação "humano+máquina", tão fortemente estudado pelas perspectivas da cognição como fenômeno de processamento da informação, para a perspectiva de coagenciamento "humano-máquina".

Os principais campos de estudo e aplicação das teorias do grupo D são as salas de controle de centrais de geração de energia, sobretudo com foco na investigação de sistemas de segurança e prevenção de acidentes. Em seus estudos de campo, ao validar o conceito de sistema cognitivo, os pesquisadores desse grupo também passam a caracterizar a cognição como um fenômeno situado, ressaltando a importância do contexto e valorizando o ambiente no qual o trabalho acontece de forma semelhante ao do grupo C (por exemplo, CHRISTOFFERSEN; WOODS, 2003; WOODS; DEKKER, 2000; WOODS, 2005; WOODS; CHRISTOFFERSEN, 2002).

No esforço de pôr em prática as premissas teóricas da cognição como um fenômeno distribuído e situado, Woods e colaboradores desenvolveram o modelo da tríade cognitiva do trabalho em contexto (Triad Cognitive Model of Work in Context) (WOODS; ROTH, 1988). A tríade representa o espaço discricionário no qual as decisões ocorrem, espaço influenciado pelos seguintes conjuntos de fatores: agentes (por exemplo, construtos mentais, conhecimentos, habilidades), as representações (a forma pela a qual o problema está estruturado) e o ambiente de trabalho (por exemplo, incerteza, dinamismo, risco, inter-relações). 
O Modelo da Tríade Cognitiva representa dois pressupostos essenciais das teorias desenvolvidas pelos pesquisadores desse grupo: (a) a ideia de que a cognição é distribuída e situada (foco no coagenciamento); (b) a valorização do contexto e do ambiente no qual o trabalho é realizado. Tais pressupostos implicam num comprometimento com a validade ecológica, em reação aos estudos de laboratório massificados pela psicologia cognitiva. Um exemplo de estudo desse grupo foram os trabalhos de Cook, Woods e Howie (1992) e Cook (1996), os quais ilustraram a forma pela qual o design de procedimentos para ajuste de válvulas controladoras de infusão automáticas aumenta a complexidade do trabalho e amplia a possibilidade da ocorrência de erros devido a imprecisão de conhecimentos e lapsos de memória, entre outros. Assim, o grupo D caracteriza-se pelo esforço de replicar as ideias segunda revolução cognitiva.

\subsection{Grupo E}

0 grupo E iniciou seus estudos na Universidade de Linköping, na Suécia, e na École des Mines de Paris, França, liderado por Erik Hollnagel e colaboradores (entre eles, D. Woods, R. Woljter e R. Cook). Os trabalhos de Hollnagel são construídos em um processo colaborativo com David Woods, líder do grupo D. Embora ambos os autores compartilhem uma série de publicações no assunto, entre as quais destacam-se as obras mais recentes Joint Cognitive System: foundations of cognitive systems engineering (HOLLNAGEL; WOODS, 2005) e Joint Cognitive System: patterns in cognitive systems engineering (WOODS; HOLLNAGEL, 2006), algumas diferenças caracterizam Hollnagel em um grupo à parte.

A abordagem do estudo da cognição de Hollnagel é também fortemente influenciada pelos teóricos da segunda revolução cognitiva. Desses estudiosos, em especial, destaca-se Ullerich Neisser, em quem o autor buscou inspiração para conceber os modelos de controle contextual (Contextual Control Model - COCOM) e estendido (Extended Control Model - ECOM) (HOLLNAGEL, 1998, 2002). Na concepção desses modelos, o autor fundamenta-se no conceito de cognição distribuída e situada (HUTCHINS, 1995b), assim como no ciclo perceptivo de Neisser (1976), para criar uma modelagem de compreensão da cognição como fenômeno de controle (GAUTHEREU; HOLLNAGEL, 2005; JOHANSSON; HOLLNAGEL, 2007; HOLLNAGEL; WOODS, 2005).

A lógica dos modelos de controle contextual e estendido posiciona o operador humano como controlador de um sistema cognitivo formado pela relação entre operador, tecnologia e o contexto operativo. Para descrever os fenômenos de controle, o autor define três elementos fundamentais dos modelos: constructos, competência e circularidades de feedback e feedforward.

Inspirados no conceito Schemata de Neisser (1976), os construtos seriam representações mentais que o operador desenvolve sobre a situação operativa. Nesse nível, ocorreriam as seleções de estratégias por parte dos operadores, podendo ser temporários ou de longa duração, aproximando-se dos conceitos de modelos mentais (HOLLNAGEL; WOODS, 2005). A ideia central é que os construtos seriam representações dos operadores constituídas pela assimilação perceptiva de informações do ambiente associadas a conhecimentos pré-existentes, por meio da ativação de recursos de memória.

0 conceito de constructo se relaciona de forma muito próxima ao conceito de competência. A competência representa o conjunto de ações ou respostas possíveis que emergem de uma situação interativa entre operador, metas da ação e contexto. Para o referido autor, as competências são capacidades estocadas no sistema cognitivo por meio de tecnologias, procedimentos padronizados e planos de contingência, definindo o escopo de possibilidades de ação do sistema.

Embora os pressupostos teóricos dos conceitos de constructos e competências, as circularidades de feedback e feedforward representam elementos centrais da lógica de controle dos modelos COCOM e ECOM. Tais circularidades são fortemente inspiradas no conceito de controle da cibernética, no qual o referido autor vai buscar embasamento nos estudos de Ashby (1959). Ao definir o controle na perspectiva da cibernética, ele aproxima o posicionamento de tais modelos aos pressupostos da primeira revolução cognitiva, imprimindo abordagens típicas das teorias de processamento da informação. Essa visão é, da mesma forma, ratificada pelo autor quando insere a noção de entropia da física na dinâmica dos sistemas cognitivos e explica o comportamento humano como agente regulador de tal sistema (HOLLNAGEL, 1998, 2002).

Mais recentemente, o autor revela seu interesse em estudar a variabilidade nos sistemas cognitivos a partir do estudo do princípio ETTO (EfficiencyThroughness Trade-Off) (HOLLNAGEL, 2009). Para ele, a variabilidade poderia ser acessada e compreendida através do uso do princípio ETTO para descrever o comportamento e o desempenho humanos.

Embora as concepções e modelos teóricos do grupo E sejam amplamente descritos na literatura sobre engenharia de sistemas cognitivos, constata-se a escassez de aplicações práticas e e validação empírica para tais pressupostos. Uma hipótese para a lacuna 
de pesquisas aplicadas utilizando tais conceitos poderia ser a falta de um protocolo descritivo para a investigação desses enunciados.

\subsection{Semelhanças e diferenças entre os grupos da ESC}

A Figura 1 apresenta uma síntese dos grupos de ESC identificados na literatura segundo: seus pesquisadores-líderes; a abordagem geral de estudo da cognição; a unidade de análise; os principais campos de atuação; e os pressupostos teóricos, modelos ou métodos desenvolvidos.

Com base na análise da Figura 1 é possivel perceber que os grupos A, B e C seguem premissas da primeira revolução científica, fortemente embasada nas teorias de processamento da informação. Já os grupos D e E possuem alinhamento com os pressupostos da segunda revolução cognitiva, considerando a cognição um fenômeno distribuído e situado. De forma complementar, os referidos grupos recorrem a categorias da primeira revolução cognitiva. Cabe ressaltar que o posicionamento aqui exposto em relação aos trabalhos do grupo $C$ vai de encontro ao proposto por Vicente (1998) e Eggleston (2002), que consideraram os pesquisadores representantes desse grupo defensores da ideia da cognição como um fenômeno distribuído e situado.

O conceito de cognição em cada grupo da ESC exerce influência na forma como os diferentes grupos definem suas unidades de análise e constituem as teorias, pressupostos e métodos. Para os grupos A e B, o conceito de cognição como algo inerente ao funcionamento da mente humana que conecta o mundo físico e o psicológico levou ao desenvolvimento de modelos que colocam o elemento humano como agente de processamento de informação do sistema.

Os pressupostos do grupo C transferem o conceito de cognição das modelagens da mente (e do comportamento humano num sentido mais amplo) para a análise de sistemas de trabalho. Ao fazer isso, os representantes desse grupo têm como unidade de análise um sistema mais amplo do que a mente, embora ainda busquem responder de que forma as dimensões físicas se conectam às dimensões psicológicas. 0 operador é, assim como nos grupos $A$ e $B$, analisado na perspectiva da resolução de problemas, identificando-se os custos cognitivos de decisões que, sempre prévias às ações, mantêm o caráter de linearidade do fluxo de ação existente nos grupos A e B.

A noção de cognição dos grupos D e E vincula-se aos pressupostos da segunda revolução científica quando considerar a cognição como fenômeno distribuído e situado, em que a unidade de análise transfere-se para os sistemas cognitivos. Nesse caso, a influência cultural e situacional das tecnologias é ressaltada. Todavia, deve-se destacar que o grupo E, embora tenha fundamentos compartilhados com o grupo D, mantém pressupostos de linearidade e análises de causa e efeito das circularidades, fortemente relacionadas ao processamento da informação e à cibernética, para a explicação dos fenômenos de controle e variabilidade.

\section{0 que as teorias e métodos em ESC estão produzindo?}

A revisão de literatura aponta que o foco da ESC está, ora no humano, ora no sistema técnico. Esta

\begin{tabular}{|c|c|c|c|c|}
\hline Grupos & $\begin{array}{c}\text { Abordagem de } \\
\text { estudo da } \\
\text { cognição }\end{array}$ & $\begin{array}{c}\text { Unidade de } \\
\text { análise }\end{array}$ & $\begin{array}{c}\text { Principais domínios de } \\
\text { aplicação }\end{array}$ & $\begin{array}{c}\text { Teorias, modelos e } \\
\text { métodos }\end{array}$ \\
\hline A & IPS & Humano & Ciência da computação & $\begin{array}{c}\text { MHP, } \\
\text { GOMS }\end{array}$ \\
\hline B & IPS & Humano & Ciência da computação & Seven-Stage Model \\
\hline C & IPS & $\begin{array}{c}\text { Sistema de } \\
\text { trabalho } \\
\text { entrais de produção de } \\
\text { saúde, ciência da de } \\
\text { computação, aviação }\end{array}$ & $\begin{array}{c}\text { Abstraction Herarquy, } \\
\text { Decision Ladder Chart, } \\
\text { SRK theory }\end{array}$ \\
\hline D & $\begin{array}{c}\text { Distribuída- } \\
\text { Situada }\end{array}$ & $\begin{array}{c}\text { Sistema } \\
\text { cognitivo }\end{array}$ & $\begin{array}{c}\text { Centrais de produção de } \\
\text { energia, sistemas de } \\
\text { saúde, aviação }\end{array}$ & JCS \\
\hline E & $\begin{array}{c}\text { IPS/Distribuída- } \\
\text { Situada }\end{array}$ & $\begin{array}{c}\text { Sistema } \\
\text { cognitivo }\end{array}$ & $\begin{array}{c}\text { Centrais de produção de } \\
\text { energia, sistemas de } \\
\text { saúde, aviação }\end{array}$ & $\begin{array}{c}\text { JCS, COCOM, ECOM, } \\
\text { ETTO }\end{array}$ \\
\hline
\end{tabular}

Figura 1. Síntese dos grupos de ESC identificados na literatura. 
seção discute implicações do foco da ESC nesses dois extremos.

\subsection{O foco no projeto do sistema técnico}

A noção de cognição como processamento da informação promoveu a ideia de que os pesquisadores poderiam identificar os mecanismos básicos do trabalho cognitivo na mente humana. Fundamentados nessa lógica, pesquisadores desenvolveram métodos e ferramentas para a identificação do fluxo de informações dentro de um sistema e os reflexos desse fluxo em seu desempenho.

Um exemplo é a estrutura de análise de Rasmussen, Pejtersen e Goodstein (1994), reinterpretada por Vicente e Rasmussen (1992) e Vicente (1999), para análise dos constrangimentos do trabalho, considerando-se o contexto, o ambiente, os artefatos e as atividades dos agentes. A referida estrutura fundamenta-se na identificação das estruturas responsáveis pelo fluxo de informação no sistema investigado. 0 desempenho, nesse caso, é baseado na qualidade do fluxo de informação e nos elementos de comunicação do próprio sistema, tais como código, canal e processo de decodificação.

A análise cognitiva das atividades nesses sistemas propicia aos pesquisadores uma compreensão dos constrangimentos do sistema. Esses constrangimentos são tipicamente tratados como pontos possíveis de intervenção para melhorias. A solução típica, segundo essa visão, é o projeto de interfaces baseadas na forma como os humanos processam informação, decidem e executam ações.

Os últimos 50 anos de estudos em psicologia cognitiva têm se caracterizado, para além de novas descobertas, pela criação de estruturas e categorias da cognição humana (por exemplo, memória, atenção, percepção). Essas categorias são "invenções científicas", enunciadas segundo pensamento vinculado à primeira revolução cognitiva, inspiradas por analogia às máquinas (sobretudo computadores), para compreender a forma como os humanos processam as informações. A consequente elevação dos humanos a categorias de processadores de informação acaba por resultar em recomendações de melhoria do sistema baseadas no processo racionalista de tomada de decisão. Dessa forma, o foco de tais melhorias acaba por resumir-se ao desenvolvimento de soluções para racionalizar o processamento de informação como um processo no sistema.

Como resultado, a visão de desempenho baseada nesse foco de investigação amplifica a necessidade de investimentos na arquitetura técnica do sistema (no hardware), a fim de facilitar o processamento da informação. Como implicações para a pesquisa em ESC, um grupo de métodos e ferramentas de investigações tem sido desenvolvido com a ênfase na qualidade do projeto e da interface técnica, tais como os métodos de Cognitive Task Design (ver HOLLNAGEL, 2003; FLACH, 1990). Os projetos de equipamentos e tarefas são, assim, legitimados como uma alternativa de solução dos problemas em sistemas complexos por meio das melhorias na qualidade do fluxo e do processamento da informação.

\subsection{O foco nos humanos}

A aplicação do conceito de cognição em ESC, seja ele influenciado pela primeira ou pela segunda revolução cognitiva, tem centrado o elemento humano como processador de informação e agente único de controle dos sistemas sociotécnicos complexos. Essa lógica tem governado os estudos dos diferentes grupos da ESC, conforme apresentado na seção anterior.

Os fenômenos de controle são investigados buscando-se, sempre, a compreensão do desempenho humano pela lógica de que existe um espaço de controle delimitado por parâmetros máximos e mínimos. Note-se que o estudo dos fenômenos da regulação em fatores humanos e ergonomia cognitiva são muito antigos e remontam às origens do estudo da cibernética (ver ASHBY, 1959). Essa lógica transpõe a ideia de controle das máquinas físicas (por exemplo, termostato) para as máquinas sociais (humanos) (VIDAL; CARVALHO, 2008).

Atenção tem sido dada ao estudo da tomada de decisão (CARD; MORAN; NEWELL, 1986; NORMAN, 1981; RASMUSSEN; PEJTERSEN; GOODSTEIN, 1994; VICENTE, 1998), da consciência situacional (ENDSLEY, 1995a, b; WICKENS, 2008), da interferência da carga de trabalho na capacidade humana de manter-se à frente das regulações do sistema (HART, 1982; HENDY et al., 2002; WIENER, 1998), da necessidade de comunicação para melhoria do fluxo cognitivo no sistema (BAKER; DISMUSKES, 2002; FLIN; O'CONNOR; CRICHTON, 2008; LETSKY et al., 2008), no gerenciamento da execução de tarefas múltiplas (LOUKOPOULOS; DISMUKES; BARSHI, 2009; MEYER; KIERAS, 1997), nos protocolos de elicitação de conhecimento (CRANDALL; KLEIN; HOFFMAN, 2006), entre outros. Como consequência, produz-se um problema de ordem do humano, pois é esse o agente central de controle do sistema. Cabe a ele o controle da variabilidade e da regulação e, logo, o modelo de controle da regulação do sistema seria reflexo do próprio modo de controle do humano dentro desse sistema (ver HOLLNAGEL, 1999, 2002, 2005).

0 foco da ESC é, pois, na maior parte das vezes, centrado no papel do humano em tais sistemas. 
Como resultado, produz-se uma verdade (com status científico) de que o humano é a parte mais frágil e imprevisível dos sistemas sociotécnicos, fonte de variabilidade e risco. Logo, os problemas de controle, as disfunções e a falhas nos sistemas sociotécnicos, quando não circunscritas exclusivamente ao hardware de tais sistemas, são sempre da ordem do humano. Tem-se aí parte do aparato que constitui a visão tradicional de erro humano e os modelos decorrentes da explicação de acidentes fortemente baseados em lógicas de linearidade, causa e efeito e controle do fluxo de energia entre "a causa" e "a consequência", por meio de barreiras (HOLLNAGEL, 2004; DEKKER, 2005a, b).

Com o foco nos humanos, essas teorias e métodos acabam por produzir um regime de verdade no qual as recomendações de melhoria de eficiência e eficácia estão sempre ligadas à necessidade de mais treinamento, mais padronização do trabalho e afastamento do humano da manipulação direta desses sistemas. 0 treinamento viria no sentido de aumentar a capacidade de controle e processamento da informação da parte humana. A padronização se justificaria para reduzir a variabilidade do comportamento humano, aumentar sua capacidade de processamento e conter a energia entrópica do sistema, segundo um repertório prescritivo de ações. 0 afastamento do humano da manipulação desses sistemas se justificaria com o foco de manter o sistema-máquina em funcionamento de forma autônoma, reduzindo a variabilidade em todo o processo de funcionamento.

Ao focar o humano como um processador de informação e controlador dentro do sistema, essa lógica acaba por produzi-lo como o responsável final pela operação e, portanto, o culpado pelas eventuais falhas. Reconhecer o humano como a parte frágil canaliza os esforços para cada vez mais investimento na expertise. Essa lógica maquínica de explicar esses sistemas produz, igualmente, a maquinização do seu operador através da normatização do trabalho e padronização dos procedimentos. 0 ato de operar e pensar um determinado sistema durante sua operação reduz-se, como consequência, ao desempenho de uma coreografia previamente determinada.

\section{A possibilidade de uma nova agenda de pesquisa}

Três premissas devem orientar uma nova agenda de pesquisa, construídas a partir da análise do posicionamento epistemológico e das produções no campo da engenharia de sistemas cognitivos. São elas: (a) um sistema cognitivo não é uma máquina cartesiano-newtoniana; (b) um sistema cognitivo não pode ser modelado com precisão e perfeição; (c) a investigação de sistemas cognitivos deve focar no acontecimento emergente da interação entre humano, trabalho e artefatos.

Um sistema cognitivo não é uma máquina cartesiano-newtoniana simplesmente porque é composto por uma dimensão social (humano) e uma dimensão técnica (tecnologia como hardware e software). As inter-relações entre essas partes não são lineares e não são completamente previsíveis. Por exemplo, a disponibilidade dos dados no contexto operativo não reflete necessariamente a observação desses dados por parte da dimensão humana e da dimensão técnica (DEKKER, 2005a; WOODS et al., 2010). Um cockpit de aeronave com o radar não sintonizado pode não capturar ou mascarar informações relativas a formações meteorológicas pesadas em rota. Da mesma forma, a capacidade de observação humana dos dados da situação operativa gera representações mentais, que são sempre parciais e incompletas. Nesse caso, humano e artefato tecnológico compõem um sistema cognitivo correlacionado que atua com representações sempre imprecisas da realidade.

A modelagem de um sistema cognitivo (ou mesmo de um sistema cognitivo correlacionado) como unidade de análise é sempre imprecisa e imperfeita (CILLIERS, 2001, 2005). Primeiramente é difícil estabelecer com precisão os limites de tais sistemas. Ao definir os limites do sistema, cria-se um conjunto de fronteiras que delimitam o que está dentro e o que está fora. 0 uso de categorias encapsula os fenômenos, primariamente, em relação aos critérios definidos à priori para essas categorias e, secundariamente, em relação à natureza dos próprios fenômenos. Consequentemente, os modelos gerados são sempre cópias imperfeitas do mundo natural. A incerteza, a variabilidade e a dinâmica estão sempre presentes, desafiando as categorias e os modelos.

Recentes trabalhos têm problematizado o estudo e a investigação de falhas em sistemas sociotécnicos, criticando tradição mecanicista cartesiano-newtoniana e sugerindo, como alternativa, o uso de teorias vinculadas à filosofia da complexidade e às ciências sociais (DEKKER, 2012; DEKKER; CELLIERS; HOFMEYR, 2011). Dekker et al. (2012), por exemplo, salientam que um sistema sociotécnico é por natureza complexo e não complicado. Sistemas complicados poderiam ser exaustivamente descritos e, portanto, modelados, pois possuem relações de causa e efeito evidentes (enunciado que seria válido para a parte técnica, não humana do sistema cognitivo). Sistemas complexos (sociotécnicos), por outro lado, não poderiam ser exaustivamente descritos nem precisamente modelados, pois possuem uma infinidade de relações não lineares e fenômenos emergentes dessas relações. 
A investigação dos sistemas cognitivos deve focar no acontecimento emergente da interação entre humanos, trabalho e artefatos (WOODS; HOLLNAGEL, 2006; WOODS et al., 2002). A emergência é uma propriedade que aparece no processo interativo de coatuação entre a parte social e a parte técnica do sistema. Tal como a junção de átomos de hidrogênio e oxigênio produzindo a água como algo totalmente novo, ela é representada por um acontecimento único, às vezes imprevisto, e constitui-se em um efeito natural do sistema (CILLIERS, 2005). Mais do que conter a emergência, instituindo práticas para lidar com a variabilidade e a incerteza, é necessário compreendê-la. Nesse sentido, mais do que focar na análise da capacidade humana de desempenhar trabalho ou focar na análise do projeto das tecnologias, é necessário um foco nos fenômenos emergentes das interações do trabalho cognitivo conjunto. Nesse sentido, o fundamental é compreender as representações ao nível do sistema cognitivo, correlacionando a natureza das interações entre os subsistemas do sistema cognitivo analisado e a cognição como algo vinculado à resiliência do sistema. Resiliência é aqui tratada na perspectiva da capacidade adaptativa de o sistema manter-se dentro de padrões normais de funcionamento, prevendo, evitando e recuperando-se de condições indesejadas de funcionamento (NEMETH; HOLLNAGEL; DEKKER, 2009).

Considerando que a lógica da maquinaria cartesiano-newtoniana pode funcionar no espaço discricionário de atuação e compreensão da máquina (hardware) mas apresenta utilidade limitada para o fenômeno social (ação do humano no sistema) e suas consequências, outras possibilidades para a investigação de sistemas cognitivos devem ser propostas. Nesse sentido, os argumentos aqui apresentados sustentam a necessidade: de estabelecerem-se critérios para a delimitação do sistema cognitivo correlacionado de análise, tomando como referência para inclusão ou não o papel dos elementos no desempenho do sistema (aquilo que influencia e aquilo que não influencia); de investigação da representação como um fenômeno emergente do processo interativo; de compreensão dos processos de interação entre os subsistemas e a emergência dos fenômenos do acontecimento. Cabe ressaltar, igualmente, que a lógica que norteia tais critérios consiste de uma produção cultural delimitada pelas circunstâncias de cada pesquisa, incorrendo em alta validade ecológica porém baixo potencial de generalização dos resultados.

\section{Considerações finais}

Este trabalho buscou, pois, realizar uma discussão do posicionamento epistemológico das pesquisas em
ESC e descrever o que tais enunciados e métodos científicos estão produzindo. Dessa maneira, foi identificado que as pesquisas em ESC vêm criando, a partir do conceito de cognição, um aparato de mecanismos científicos para a compreensão do desempenho dos sistemas cognitivos. Alguns grupos das pesquisas em ESC vinculam o conceito de cognição à teoria de processamento da informação; outros grupos vinculam ao conceito de cognição distribuída e situada. De qualquer forma, as pesquisas em ESC possuem focos permanentes no humano e na máquina, estando, ainda, fortemente vinculadas à ideia de produção de modelos.

0 pensamento cartesiano-newtoniano tem influenciado e limitado a compreensão mais ampla dos sistemas cognitivos devido à busca desenfreada pela compreensão das estruturas e do funcionamento dos dispositivos (aquilo que coloca em funcionamento a operacionalização de um conceito nessa maquinaria) implícitos ao desempenho desses sistemas. Nessa visão, a lógica exata de engenharia e reengenharia da máquina tem sido utilizada para a compreensão de fenômenos sociotécnicos mais complexos tais como o desempenho, as falhas e os "erros humanos".

Se, por um lado, a psicologia cognitiva experimental buscou abrir a "caixa preta" da mente humana para compreender o processamento mental da informação e com isso auxiliar na explicação dos comportamentos humanos, por outro lado o estudo da ESC tem se mostrado como uma volta ao behaviorismo ao reposicionar a unidade de análise do humano como um processador de informações e controlador de um sistema para a própria condição do sistema como unidade de análise. A caixa preta passou a ser o sistema cognitivo. Não se trata de saber se é verdadeiro ou falso, mas como essas teorias com força de verdade estão produzindo a realidade na qual se desenvolvem categorias para explicar esse sistema.

Por fim, cabe ressaltar que o próprio conceito de complexidade pode ser perspectivado. Resume-se a complexidade a categorias como variabilidade, incerteza, dinamicidade e risco, as quais representam os pontos fundamentais de ruptura na lógica cartesiano-newtoniana, quando operacionalizadas. Mas, dialeticamente, a complexidade só existe em um mundo governado pela noção de ciência cartesiana-newtoniana.

\section{Referências}

ALBRECHTSEN, $\mathrm{H}$. et al. Affordance in activity theory and cognitive systems engineering (RISO-r-1287(EN)). Roskield: RISO National Laboratory, 2001.

ASHBY, W. R. Requisite variety and its implications for the control of complex systems. Cybernetica, v. 1, p. 83-99, 1959.

BAKER, D.; DISMUKES, R. A Framework for Understanding Crew Performance Assessment 1ssues. International Journal of Aviation Psychology, v. 12, n. 3, p. 205-222, 2002. http://dx.doi.org/10.1207/S153271081JAP1203_2 
BAARS, B. J. The cognitive revolution in psychology. New York: Guilford Press, 1986.

BEM, S.; KEIJZER, F. Recent changes in the concept of cognition. Theory and Psychology, v. 6, p. 449-469, 1996. http://dx.doi.org/10.1177/0959354396063006

BISANTZ, A. M.; VICENTE, K. J. Making the abstraction hierarchy concrete. International Journal of HumanComputer Studies, v. 40, n. 1, p. 83-117, 1994. http:// dx.doi.org/10.1006/ijhc.1994.1005

BOVAIR, S.; KIERAS, D. E.; POLSON, P. G. The acquisition and performance of text editing skill: A cognitive complexity analysis. Human-Computer Interaction, v. 5, p. 1-48, 1990. http://dx.doi.org/10.1207/ s15327051hci0501_1

BURNS, K. Improving Intuition with Bayesian Boxes: On Cognitive Difficulties in Combining Probabilities. In: HOFFMAN, R. (Ed.). Expertise out of the context. Proceedings of the Sixth International Conference on Naturalistic Decision Making. New York: Lawrence Erlbaum Associates, 2007. p. 263-280.

CARD, S. K.; MORAN, T. P.; NEWELL, A. The keystroke-level model for user performance time with interactive systems. Communications of the ACM, v. 23, n. 7, p. 396-410, 1980. http://dx.doi.org/10.1145/358886.358895

CARD, S. K.; MORAN, T. P.; NEWELL, A. The psychology of computer-human interaction. Hillsdale: Erlbaum, 1983.

CARD, S. K.; MORAN, T. P.; NEWELL, A. The model human processor: An engineering model of human performance. ln: BOFF, K.; KAUFMAN, L.; THOMAS, J. (Eds.). Handbook of perception and human performance, Vol ll. Cognitive Processes and Performance. New York: Wiley, 1986.

CILlIERS, P. Boundaries, Hierarchies and Networks in Complex Systems. International Journal of Innovation Management, v. 5, n. 2, p. 135-147, 2001.

CILLIERS, P. Complexity, Deconstruction and Relativism. Theory Culture \& Society, v. 22, n. 5, p. 255-267, 2005. http://dx.doi.org/10.1177/0263276405058052

CHRISTOFFERSEN, K.; WOODS, D. D. Making sense of change: Extracting events from dynamic process data. Columbus: The Ohio State University, 2003. Institute for Ergonomics/Cognitive Systems Engineering Laboratory Report ERG0-CSEL 01-TR-02.

COOK, R. l. Adapting to the new technology in the operating room. Human Factors, v. 38, n. 4, p. 593-613, 1996. http://dx.doi.org/10.1518/001872096778827224

COOK, R. 1.; WOODS, D. D.; HOWIE, M. B. Unintentional delivery of vasoactive drugs with an electromechanical infusion device. Journal of Cardiothoracic and Vascular Anesthesia, v. 6, p. 1-7, 1992. http://dx.doi. org/10.1016/1053-0770(92)90207-N

CRANDALL, B.; KLEIN, G.; HOFFMAN, R. Working Minds: a practitioner's guide to cognitive task analysis. Cambridge: MIT Press, 2006.

DEKKER, S. W. A. Crew situation awareness in hightech settings: Tactics for research into an ill-defined phenomenon. Transportation Human Factors, v. 2, p. 49-62, 2000. http://dx.doi.org/10.1207/STHF0201_7

DEKKER, S. W. A. Ten questions about human error. Aldershot: Ashgate, 2005a.

DEKKER, S. W. A. Why we need new accident models? Lund: Lund University School of Aviation, 2005b. Technical Report 2005-02.

DEKKER, S. W. A. Complexity, signal detection, and the application of ergonomics: reflections on a healthcare study.
Journal of Applied Ergonomics, v. 43, p. 468-472, 2012. http://dx.doi.org/10.1016/j.apergo.2011.07.003

DEKKER, S. W. A. et al. Complicated, Complex and Compliant: best practices in obstetrics. Cognition, Technology, and Work. 2012. In press. http://dx.doi.org/10.1007/s10111011-0211-6

DEKKER, S. W. A.; CELlIERS, F. P.; HOFMEYR, J. H. The complexity of failure: implications of complexity theory for safety investigations. Safety Science, v. 49, n. 6, p. 939-945, 2011. http://dx.doi.org/10.1016/j. ssci.2011.01.008

DEKKER, S. W. A.; HOLLNAGEL, E. Human factors and folk models. Cognition, Technology, and Work, v. 6, p. 79-86, 2004. http://dx.doi.org/10.1007/s10111-0030136-9

DEKKER, S. W. A. et al. Epistemological self-confidence in human factors research. Journal of Cognition and Decision Making, v. 4, n. 1, p. 27-38, 2010. http://dx.doi. org/10.1518/155534310X495573

DEKKER, S. W. A.; WOODS, D. D. MABA-MABA or abracadabra? Progress on human automation coordination. Cognition, Technology, and Work, v. 4, p. 40-244, 2002. http:// dx.doi.org/10.1007/s101110200022

EGGLESTON, R. G. Cognitive systems engineering at 20-something: where do we stand? In: McNEESE, M. D.; VIDULICH, M. A. (Eds.). State of the art report (SOAR): Cognitive systems engineering in military aviation environments: Avoiding cogminutia fragmentosa. Wright-Patterson AFB: Human Systems Information Analysis Center, 2002. p. 13-77.

ENDSLEY, M. R. Toward a theory of situation awareness in dynamic systems. Human Factors, v. 37, p. 32-64, 1995a. http://dx.doi.org/10.1518/001872095779049543

ENDSLEY, M. R. Measurement of situation awareness in dynamic systems. Human Factors, v. 37, p. 65-84, 1995 b. http://dx.doi.org/10.1518/001872095779049499

ENDSLEY, M. R.; KABER, D. B. Level of automation effects on performance, situation awareness and workload in a dynamic control task. Ergonomics, v. 42, p. 462-492, 1999. http://dx.doi.org/10.1080/001401399185595

ENDSLEY, M. R.; KIRIS, E. 0. The out-of-the-loop performance problem and level of control in automation. Human Factors, v. 37, p. 381-394, 1995. http://dx.doi. org/10.1518/001872095779064555

ENDSLEY, M. R.; GARLAND, D. J. (Eds.). Situation Awareness Analysis and Measurement. Mahawah: Lawrence Erlbaum Associates, 2000.

ENDSLEY, M. R. et al. Cognitive engineering and decision making: an overview and future course. Journal of Cognitive Engineering and Decision Making, v. 1, n. 1, p. 1-21, 2007. http://dx.doi.org/10.1177/155534340700100101

FEDERAL AVIATION ADMINISTRATION - FAA. Aeronautical Decision Making. Advisoty Circular 60-22. Washington: Federal Aviation Administration, 1991.

FLACH, J. M. The ecology of human-machine systems 1: Introduction. Ecological Psychology, v. 2, n. 3, p. 191-205, 1990. http://dx.doi.org/10.1207/ s15326969eco0203_1

FLIN, R.; O'CONNOR, P.; CRICHTON, M. Safety at the sharp end: a guide to non-technical skills. Burlington: Ashgate, 2008.

GAUTHEREU, V.; HOLLNAGEL, E. Planning, control and adaptation. European Management journal, v. 23, n. 1, p. 118-131, 2005. http://dx.doi.org/10.1016/j. emj.2004.12.016 
GlBBSON, J. J. The ecological approach to visual perception. Boston: Houghton-Mifflin, 1979.

GRAY, W. D.; JOHN, B. E.; ATWOOD, M. E. The Précis of Project Ernestine or an overview of a validation of GOMS. In: BAUERSFELD, P.; BENNETT, J.; LYNCH, G. (Eds.). Proceedings of the ACM CHl'92 Conference on Human factors in Computing Systems. New York: ACM Press, 1992. p. 307-3012.

HART, S. G. Theoretical basis for workload assessment research at NASA - Armes Research Center. Defense Technical Information Center, 1982. p. 445-470. Proceedings of the workshop on flight testing to identify pilot workload and pilot dynamics (AFTECTR-82-5).

HARRÉ, R. The Rediscovery of the human mind: The discursive approach. Asian Journal of Social Psychology, v. 2, p. 43-62, 1999. http://dx.doi.org/10.1111/1467839X.00025

HATFIELD, G. Psychology, philosophy, and cognitive science: Reflections on the history and philosophy of experimental psychology. Mind \& Language, v. 17, p. 207-232, 2002. http://dx.doi.org/10.1111/1468-0017.00196

HENDY, K. C. et al. Analysing cognitive system from a perceptual control point of view. In: McNEESE, M. D.; VIDULICH, M. A. (Eds.). State of the art report (SOAR): Cognitive systems engineering in military aviation environments: Avoiding cogminutia fragmentosa. Wright-Patterson AFB: Human Systems Information Analysis Center, 2002. p. 201-250.

HICK, W. E. On the rate of gain of information. Quarterly Journal of Experimental Psychology, v. 4, p. 11-26, 1952. http://dx.doi.org/10.1080/17470215208416600

HYMAN, R. Stimulus information as a determinant of reaction time. Journal of Experimental Psychology, v. 45, p. 188-196, 1953. http://dx.doi.org/10.1037/h0056940

HOFFMAN, R. et al. A rose by any other name...would probably be given an acronym. IEEE Inteligent Systems, v. 17, n. 4, p. 72-80, 2002. http://dx.doi.org/10.1109/ MIS.2002.1024755

HOFFMAN, R.; WOODS, D. D. Special Section on Studying Cognitive Systems in Context. Human Factors, v. 42, n. 1, p. 1-7, 2000. http://dx.doi. org/10.1518/001872000779656633

HOLLNAGEL, E. Context, cognition, and control. In: WAERN, Y. (Ed.). Co-operative in process management: Cognition and information technology. London: Taylor \& Francis, 1998. p. 27-51.

HOLLNAGEL, E. Modeling the controller of a process. Transactions of the Institute of Measurement and Control, v. 21, n. 4-5, p. 163-170, 1999. http://dx.doi. $\operatorname{org} / 10.1177 / 014233129902100404$

HOLLNAGEL, E. Time and Time Again. Theoretical Issues in Ergonomic Science, v. 3, n. 2, p. 143-158, 2002. http:// dx.doi.org/10.1080/14639220210124111

HollNAGEL, E. Prolegomenon to Cognitive Task Design. In: HOLLNAGEL, E. (Ed.). Handbook of Cognitive Task Design. Mahawah: Lawrence Erlbaum Associates, 2003. p. 03-16. http://dx.doi.org/10.1201/9781410607775

HOLLNAGEL, E. Barriers and accident prevention. Aldershot: Ashgate Publishing, 2004.

HOLLNAGEL, E. Human reliability assessment in context. Nuclear Engineering and Technology, v. 37, n. 2, p. 159-166, 2005.

HOLlNAGEL, E. The ETTO Principle Efficiency-Thoroughness Trade-Off. Surrey: Ashgate Publishing Company, 2009.
HOLLNAGEL, E.; WOODS, D. D. Cognitive systems engineering: New wine in new bottles. International Journal of ManMachine Studies, v. 18, p. 583-600, 1983. http://dx.doi. org/10.1016/S0020-7373(83)80034-0

HOLlNAGEL, E.; WOODS, D. D. Joint Cognitive Systems: foundations of cognitive systems engineering. Boca Raton: Taylor \& Francis, 2005. http://dx.doi. org/10.1201/9781420038194

HUTCHINS, E. How a cockpit remembers its speeds. Cognitive Science, v. 19 , p. 265-288, 1995a. http://dx.doi. org/10.1207/s15516709cog1903_1

HUTCHINS, E. Cognition in the wild. Cambridge: MIT Press, 1995b.

JOHANSSON, B.; HOLLNAGEL, E. Pre-requisites for large scale coordination. Cognition, Technology, and Work, v. 9, p. 5-13, 2007. http://dx.doi.org/10.1007/s10111006-0050-z

JOHNSON-LAIRD, P. N. Mental models in cognitive science. Cognitive Science, v. 4, p. 7-115, 1980. http://dx.doi. org/10.1207/s15516709cog0401_4

KABER, D. B.; ENDSLEY, M. R. The effects of level of automation and adaptive automation on human performance, situation awareness and workload in a dynamic control task. Theoretical Issues in Ergonomics Science, v. 5, n. 2, p. 113-153, 2004. http://dx.doi. org/10.1080/1463922021000054335

KABER, D. B.; RILEY, J. Adaptive automation of a dynamic control task based on secondary task workload measurement. International Journal of Cognitive Ergonomics, v. 3, n. 3, p. 169-187, 1999. http://dx.doi. org/10.1207/s15327566ijce0303_1

KIERAS, D. E. Toward a practical GOMS model methodology for user interface design. In: HELANDER, M. (Ed.). The handbook of human-computer interaction. Amsterdam: North-Holland, 1988. p. 135-158.

KIERAS, D. E. A guide to GOMS model usability evaluation using NGOMSL. 1996. Disponivel em: <http://www. ftp.eecs.umich.edu people/kieras>. Acessado em: 15 Maio 2010.

KIERAS, D. E.; BOVAIR, S. The role of a mental model in learning to operate a device. Cognitive Science, v. 8 , p. 255-273, 1984. http://dx.doi.org/10.1207/ s15516709cog0803_3

KIERAS, D. E.; POLSON, P. G. An approach to the formal analysis of user complexity. International Journal of Man-Machine Studies, v. 22, p. 365-394, 1985. http:// dx.doi.org/10.1016/S0020-7373(85)80045-6

LASZLO, J. Narrative psychology's contribution to second cognitive revolution. Journal of Cultural and Evolutionary Psychology, v. 2, p. 337-354, 2004. http:// dx.doi.org/10.1556/JCEP.2.2004.3-4.10

LETSKY, M. P. et al. (Eds.). Macrocognition in teams: theories and methodologies. Aldershot: Ashgate, 2008.

LOPES, E. J.; LOPES, R.; TEIXEIRA, R. F. A psicologia cognitiva experimental cinquenta anos depois: a crise do paradigma do processamento de informação. Paidea, v. 14, n. 27, p. 17-26, 2004.

LOUKOPOULOS, L. D.; DISMUKES, R. K.; BARSHI, I. The multitasking myth: Handling complexity in real-world operations. Aldershot: Ashgate Publishing Co, 2009.

McCLELLAND, J. L. On the time relations of mental processes. An examination of processes in cascade. Psychological Review, v. 86, p. 287-330, 1979. http://dx.doi. org/10.1037/0033-295X.86.4.287 
McCULLOCH, W.; PITTS, W. A logical calculus of the ideas immanent in nervous activity. Bulletin of Mathematical Biophysics, v. 5, p. 15-133, 1943. http://dx.doi. org/10.1007/BF02478259

McNEESE, M. D. Discovering how cognitive systems engineering should be engineered for aviation domains: a developmental look at work, research, and practice. In: McNEESE, M. D.; VIDULICH, M. A. (Eds.). State of the art report (SOAR): Cognitive systems engineering in military aviation environments: Avoiding cogminutia fragmentosa. Wright-Patterson AFB: Human Systems Information Analysis Center, 2002. p. 79-119.

MEYER, D. E.; KIERAS, D. E. A computational theory of executive control processes and human multiple task performance: Part 1: Basic mechanisms. Psychological Review, v. 104, p. 3-65, 1995. http://dx.doi. org/10.1037/0033-295X.104.1.3

MILLER, J. Discrete and continuous models of human information processing: Theoretical distinctions and empirical results. Acta Psychologica, v. 67, p. 191-257, 1998. http://dx.doi.org/10.1016/00016918(88)90013-3

MILLER, G. A. The cognitive revolution: A historical perspective. Trends in Cognitive Sciences, v. 7, p. 141-144, 2003. http://dx.doi.org/10.1016/S1364-6613(03)00029-9

NAIKAR, N.; SANDERSON, P. M. Work domain analysis for training-system definition and acquisition. International Journal of Aviation Psychology, v. 9, n. 3, p. 271-290, 1999. http://dx.doi.org/10.1207/ s15327108ijap0903_5

NAIKAR, N.; SANDERSON, P. M.; LINTERN, G. F/A-18 work domain analysis: identification of training needs for simulator acquisition. Melbourne: Aeronautical and Maritime Research Laboratory, 2000. DSTO Client Report, DSTO-CR-0117.

NEISSER, U. Cognitive psychology. New York: AppletonCentury-Crofts, 1967.

NEISSER, U. Cognition and Reality. San Francisco: W. H. Freeman, 1976.

NEMETH, C.; HOLLNAGEL, E.; DEKKER, S. W. A. (Eds.). Resilience engineering perspectives, Volume 2: preparation and restoration. Burlington: Ashgate, 2009.

NEWELL, A. Physical symbol systems. Cognitive science, v. 4, p. 135-183, 1980. http://dx.doi.org/10.1207/ s15516709cog0402_2

NEWELL, A.; SHAW, J. C.; SIMON, H. A. Elements of a theory of human problem solving. Psychological Review, v. 65, p. 151-166, 1958. http://dx.doi.org/10.1037/h0048495

NORMAN, D. A. Steps towards a cognitive engineering. San Diego: University of California, Program in Cognitive Science, 1981. Technical Report.

NORMAN, D. A. Cognitive engineering. In: NORMAN, D. A.; DRAPER, S. W. (Eds.). Usercentered system design. Mahwah: Erlbaum, 1986. p. 31-61.

O'DONOHUE, W.; FERGUSON, K. E.; NAUGLE, A. E. The structure of the cognitive revolution: an examination from the philosophy of science. The Behavior Analyst, v. 26, p. 85-110, 2003.

OLSON, J. R.; OLSON, G. M. The growth of cognitive modeling in human-computer interaction since GOMS. Human-Computer Interaction, v. 5, p. 221-265, 1990. http://dx.doi.org/10.1207/s15327051hci0502\&3_4

PARASURAMAN, R.; RILEY, V. Humans and automation: Use, misuse, disuse and abuse. Human
Factors, n. 39, p. 230-253, 1997. http://dx.doi. org/10.1518/001872097778543886

PARASURAMAN, R.; SHERIDAN, T. B.; WICKENS, C. D. A model of types and levels of human interaction with automation. IEEE Transactions on Systems, Man and Cybernetics, n. 30, p. 286-297, 2000.

PARASURAMAN, R.; SHERIDAN, T. B.; WICKENS, C. D. Situation awareness, mental workload and trust in automation: Viable empirically supported cognitive engineering constructs. Cognitive Engineering and Decision Making. Journal of Cognitive Engineering and Decision Making, v. 2, p. 140-160, 2008. http://dx.doi. org/10.1518/155534308X284417

PIAGET, J. Estudos Sociológicos. Rio de Janeiro: Forense, 1973.

POTTER, S. S. et al. Bridging the gap between cognitive analysis and effective decision aiding. In: McNEESE, M. D.; VIDULICH, M. A. (Eds.). State of the art report (SOAR): Cognitive systems engineering in military aviation environments: Avoiding cogminutia fragmentosa. Wright-Patterson AFB: Human Systems Information Analysis Center, 2002. p. 137-168.

POSNER, M. 1.; DEHAENE, S. Attentional networks. Trends in neurosciences, v. 17, p. 75-79, 1994. http://dx.doi. org/10.1016/0166-2236(94)90078-7

POSNER, M. l. et al. Localization of cognitive operations in the human brain. Science, v. 240, p. 1627-1631, 1988. http://dx.doi.org/10.1126/science.3289116

RASMUSSEN, J. Trends in human reliability analysis. Ergonomics, v. 28, n. 8, p. 1185-1195, 1985. http:// dx.doi.org/10.1080/00140138508963241

RASMUSSEN, J. Information processing and human-machine interaction; an approach to cognitive engineering. New York: Elsevier Science Publishing, 1986.

RASMUSSEN, J.; PEJTERSEN, A. M.; GOODSTEIN, L. P. Cognitive systems engineering. New York: Wiley, 1994.

RASMUSSEN, J.; PEJTERNSEN, A. M.; SHMIDT, K. Taxonomy for Cognitive Work Analysis. Roskield: RISO National Laboratory, 1990.

REASON, J. Human error. Cambridge: Cambridge University Press, 1990. http://dx.doi.org/10.1017/CB09781139062367

RESING, J. M. Cognitive engineering and its relation to future aviation systems. In: McNEESE, M. D.; VIDULICH, M. A. (Eds.). State of the art report (SOAR): Cognitive systems engineering in military aviation environments: Avoiding cogminutia fragmentosa. Wright-Patterson AFB: Human Systems Information Analysis Center, 2002. p. 1-12.

SARTER, N. B.; AMALBERTI, R. (Eds.). Cognitive Engineering in the Aviation Domain. Mahwah: Lawrence Erlbaum, 2005.

SKINNER, B. F. The origins of cognitive thought. American Psychologist, v. 44, p. 13-18, 1989. http://dx.doi. org/10.1037/0003-066X.44.1.13

SPERBER, D.; HIRSCHFELD, L. Culture, cognition, and evolution. In: WILSON, R. A. KEIL, F. C. (Eds.). The MIT encyclopedia of the cognitive sciences. Cambridge: MIT Press, 2001.

VICENTE, K. J. Commentary on evolutionary perspective on the growth of cognitive engineering: the Riso genotype. Ergonomics, v. 41, n. 2, p. 156-159, 1998. http://dx.doi. org/10.1080/001401398187189

VICENTE, K. J. Cognitive work analysis: Towards safe, productive and healthy computer-based work. Mahwah: Erlbaum, 1999. 
VICENTE, K. J.; RASMUSSEN, J. Ecological interface design: Theoretical Foundations. IEEE Transactions on Systems, Man, and Cybernetics, v. 22, n. 4, p. 589-606, 1992. http://dx.doi.org/10.1109/21.156574

VIDAL, M. C.; CARVALHO, P. V. R. Ergonomia Cognitiva: raciocínio e decisão no trabalho. Rio de Janeiro: EVC Editora, 2008.

VYGOTSKY, L. Mind in Society. The Development of Higher Psychological Processes. Cambridge: Harvard University Press, 1978.

WAGEMANS, J.; VERSTRATEN, F. A. J.; HE, S. Beyond the decade of the brain: towards a functional neuroanatomy of the mind. Acta Psychologica, v. 107, p. 1-7, 2001. http://dx.doi.org/10.1016/S0001-6918(01)00040-3

WICKENS, C. D. Engineering psychology and human performance. 2nd. New York: Harper Collins, 1992.

WICKENS, C. D. Situation Awareness: review of Mica Endsley's 1995 article on situation awareness theory and measurement. Human Factors, v. 50, p. 397-403, 2008. http://dx.doi.org/10.1518/001872008X288420

WICKENS, C.; HOLLAND, J. G. Engineering Psychology and Human Performance. Englewood Cliffs: PrenticeHall, 2000.

WIENER, E. L. Cockpit automation. In: WIENER, E. L.; NAGEL, D. C. (Eds.). Human factors in aviation. San Diego: Academic, 1988. p. 433-461.

WIENER, E. L.; CURRY, R. E. Flight deck automation: Promises and problems. Ergonomics, v. 23, p. 995-1011, 1980. http://dx.doi.org/10.1080/00140138008924809

WOODS, D. D. Process-Tracing Methods for the Study of Cognition Outside of the Experimental Psychology Laboratory. In: KLEIN, G. A. et al. (Eds.). Decision Making in Action: Models and Methods. Mahwah: Ablex Publishing Corporation, 1993.

WOODS, D. D. Discovering how distributed cognitive systems works. In: HOLLNAGEL, E. (Ed.). Handbook of Cognitive Task Design. Hillsdale: Lawrence
Erlbaum Associates, 2003. p. 37-54. http://dx.doi. org/10.1201/9781410607775.ch3

WO0DS, D. D. Creating foresight: lessons for enhancing resilience from Columbia. In: STARBUCK, W.H.; FARJOUN, M. (Eds.) Organization at the limit: lessons from the Columbia Disaster. Malden: Blackwell Publishing, 2005. p.289-308.

WOODS, D. D.; CHRISTOFFERSEN, K. Balancing practicecentered research and design. In: McNEESE, M. D. VIDULICH, M. A. (Eds.). State of the art report (SOAR): Cognitive systems engineering in military aviation environments: Avoiding cogminutia fragmentosa Wright-Patterson AFB: Human Systems Information Analysis Center, 2002. p. 121-134.

WOODS, D. D.; DEKKER, S. W. A. Anticipating the Effects of Technological Change: A New Era of Dynamics for Human Factors. Theoretical Issues in Ergonomic Science, v. 1, n. 3, p. 272-282, 2000. http://dx.doi. org/10.1080/14639220110037452

W00DS, D. D. et al. Behind human error. Aldershot: Ashgate Publishing Co, 2010.

WOODS, D. D.; HOLLNAGEL, E. Joint cognitive systems: patterns in cognitive systems engineering. Boca Raton: Taylor \& Francis, 2006. http://dx.doi. org/10.1201/9781420005684

WOODS, D. D.; ROTH, E. M. Cognitive engineering: Human problem solving with tools. Human Factors, v. 30, p. 415-430, 1988.

WOODS, D. D. et al. Studying cognitive work in context: Facilitating insight at the intersection of people, technology and work. Cogn. Syst. Eng. Lab. Inst. Ergon.; Ohio State University, Columbus, 2002. Disponível em: $<$ http://csel.eng.ohio-state.edu/woodscta>. Acessado em: 15 dez. 2008.

YERKES, R. M.; DODSON, J. D. The relation of strength of stimulus to rapidity of habit-formation. Journal of Comparative and Neurological Psychology, v. 18, p. 459482, 1908. http://dx.doi.org/10.1002/cne.920180503

\title{
Analysis of the conceptual field of cognitive systems engineering and the proposal of a new research agenda
}

\begin{abstract}
This article aims to describe the evolution of the conceptual field of cognitive systems engineering (CSE), to discuss its epistemological foundations and to verify the coherence of CSE with the nature of the problems that are investigated. Toward this aim, this work presents a literature review of the historical development of CSE and characterizes five common approaches in CSE research. The results indicate that studies in CSE have, as a rule, assumed the individual or the technical system to be the unit of analysis, ignoring important opportunities in the investigation of the interactions among human, work, and artifacts. A new research agenda is proposed based on an analysis of three assumptions: a cognitive system should not be assumed to be a Cartesian-Newtonian machine, which has clear cause-and-effect relationships; a cognitive system cannot be modeled with precision and perfection; and the investigation of cognitive systems should focus on emergent phenomena related to the interactions among human, work, and technological artifacts.
\end{abstract}

\section{Keywords}

Cognitive systems engineering. Complex socio-technical systems. Possibilities for a research agenda. 